\title{
Electronic and Optical Properties of SiGe alloys within first-principles schemes
}

\author{
G. Cappellini*, G. Satta*, M. Palummo**, G. Onida*** \\ *SLACS-INFM and Dipartimento di Fisica, Università di Cagliari, I-09042 \\ Monserrato (Cagliari), Italy \\ **INFM and Dipartimento di Fisica, II Università di Roma "Tor Vergata", \\ I-00133 Rome, Italy \\ ***INFM and Dipartimento di Fisica, Università di Milano, I-20133 \\ Milano, Italy
}

\begin{abstract}
We present first-principles calculated electronic and optical properties of some SiGe alloys. The ground-state, electronic excitations and optical properties have been calculated with Ge and Si atoms arranged in different ways among the sites of a diamond-type lattice. For the ground state a DFT-LDA scheme and for the electronic excitations a DFT-GW approach have been respectively used. For the optical properties the DFT-LDA-RPA scheme has been applied for alloys going in composition from $\mathrm{Si}(100 \%)$ to $\mathrm{Ge}(100 \%)$ : obtained results have been compared with existing experimental and theoretical data. For the noticeable $\mathrm{Si}(50 \%) \mathrm{Ge}(50 \%)$ alloy also two-particle effects have been evaluated using the Bethe-Salpeter equation.
\end{abstract}

\section{INTRODUCTION}

The elemental semiconductors silicon and germanium form a continuous series of disordered alloys. A detailed comprehension of the properties of the Si-Ge alloys is a prerequisite for successful device applications. These alloys seem promising candidates as substitute material for Si in low-power and high speed semiconductor devices[1]. Interesting properties range from enhanced mobility [2] to appearance of new direct optical transitions[3].Si-Ge semiconductors are being used to create high-performance bipolar transistors and integrated circuits $[4,5]$, highly integrated focal-plane array infrared detectors[6], and infrared light-emitting diodes [7]. The wide range of applications of Si-Ge alloys have been presented nowadays also to a wider public [8]. In this paper, we concentrate on the bulk properties of Si-Ge alloys. We determine for these systems, with state of the art computational techniques, 
the ground state, the electronic excitations and the optical absorption, going in composition from $\mathrm{Si}(100 \%)$ to $\mathrm{Ge}(100 \%)$. Obtained results have been compared with existing experimental and theoretical data.

\section{MODEL AND STRUCTURAL PROPERTIES}

In the present paper we mimic SiGe alloys by a series of ordered crystals formed from Ge and Si atoms arranged in different ways among the sites of a diamond-type lattice. Since a diamond-type lattice is composed of eight interprenetating simple cubic lattices, it is convenient to work with a series of ordered crystals having in compositions $\mathrm{Ge}_{k} \mathrm{Si}_{1-k}$ where $k=0,1 / 8, \ldots, 8 / 8$ [9]. In this way we can simulate ordered alloys with compositions steps of $12.5 \%$. The simulation of ordered structures permits us to takle the study of the SiGe alloys using small simulation cells, with a relative high number of symmetries, mantaining therefore the same efficiency of all the computational schemes which treat ground-state, electronic-excitation and optical properties. We use Density Functional Theory (DFT) in the Local Density Approximation (LDA) for the exchange and correlation energy [10].

Plane waves expansion for the wave-function with energy cut-off of 55 Rydberg, norm-conserving Troullier-Martins ionic pseudopotentials[11], from 8 to $10 \mathrm{k}$-points in the first Brillouin Zone (ISTBZ) have been used to determine the ground state of each different alloy here considered. The theoretical lattice parameters for the different structures have been then used for optical absorption spectra calculation (e.g. for $\mathrm{Ge}(100 \%), \operatorname{Si}(50 \%) \mathrm{Ge}(50 \%), \mathrm{Si}$ $(100 \%)$ they are respectively 10.57, 10.36, 10.2 a.u.).

\section{ELECTRON EXCITATION AND OPTICAL PROPERTIES}

For the discussion of the electronic properties of the SiGe alloys we consider in details the $\mathrm{Si}(50 \%) \mathrm{Ge}(\% 50)$ as prototype case. As found by other authors this is an indirect gap semiconductor with transition along the $\Delta$ line of the ISTBZ. Within LMTO Schmid and coworkers found a value of $1.22 \mathrm{eV}$ at $087 \Delta[12]$, Theodorou and coworkers found $0.82 \mathrm{eV}$ within a tight-binding approach at $0.85 \Delta[13]$, while Remediakis and Kaxiras found $1.20-1.24 \mathrm{eV}$ [14]. In the present case we find a DFT-LDA minimum gap along $\Delta$ of $0.55 \mathrm{eV}$. Due to the fact that DFT-LDA electronic energies suffer of the socalled band-gap underestimate[15, 10], one has to use another scheme (called DFT-GW), which treats correctly self-energy effects, to obtain results which compare well with experiments. DFT-GW method gives for example a selfenergy correction for the minimum gap of bulk Si of $0.77 \mathrm{eV}$. In the case 
of $\mathrm{Si}(50 \%) \mathrm{Ge}(\% 50)$ a correction slightly smaller here results, i.e. $0.57 \mathrm{eV}$, giving a minimun indirect gap of $1.12 \mathrm{eV}$ in good accordance with the data Ref.[12, 14].

In principle state of the art calculations of the optical properties of semiconductors should include the one-particle and two-particle effects [10]. However it has been demonstrated that in optical absorption spectra of many semiconductors these two effects nearly cancel, therefore making the DFTLDA dielectric function calculated within the random-phase approximation (RPA) a valuable term of comparison with experiments [10, 11]. Moreover in the case of the electron-energy loss spectra one has to evaluate the function $-\operatorname{Im}\left[\epsilon^{-1}(\omega)\right]$ which determined within DFT-LDA-RPA scheme gives very good comparison with the experiments[10]. Another point in favour of the DFT-LDA-RPA scheme is that the computational limits of the BetheSalpeter Equation (BSE)approach, which takes properly into account the electron-hole interaction, poses much more difficulties to its straight application to medium and large size systems. For these reasons within the present paper we are able to treat properly the one- and two-particle effects only in pure $\mathrm{Si}$, pure $\mathrm{Ge}$ and in the relevant case of $\mathrm{Si}(50 \%) G e(50 \%)$. This alloy is particularly interesting because it has the average composition between the two constituents, and it can be seen as the prototype for all $(\mathrm{Ge})_{n} /(\mathrm{Si})_{m}[12]$ superlattices. The understanding of $\mathrm{Si} / \mathrm{Ge}$ superlattices is particularly appealing because of the possibility of obtaining a direct or Si-based quasi-direct semiconductor material[12]. Moreover the possibility of comparison between DFT-LDA-RPA and BSE schemes permits one to test the efficiency of the DFT-LDA-RPA scheme which is the only that at the moment can be applied to medium and large size system (in this case to all the alloys with composition different from $\mathrm{Si}(100 \%), \mathrm{Ge}(100 \%), \mathrm{Si}(50 \%) \mathrm{Ge}(50 \%))$. In the reciprocal space the microscopic dielectric matrix within DFT-LDA-RPA reads[15]

$$
\epsilon_{\vec{G}, \overrightarrow{G^{\prime}}}^{R P A}(\vec{q}, \omega)=1-v(\vec{q}+\vec{G}) \chi_{\vec{G}, \vec{G}^{\prime}}^{R P A}(\vec{q}, \omega) .
$$

in which $\chi_{\vec{G}, \vec{G}^{\prime}}^{R P A}(\vec{q}, \omega)$ is the RPA susceptibility tensor, which reads as product of two one-particle Green functions $[10,15]$. In the present work we determine the above nonlocal function from DFT-LDA eigenvalues and eigenvectors. Due to the need of good convergence for optical calculations[10], all the optical functions here given have been converged with a $512 \vec{k}$-points mesh in ISTBZ. In Fig. 1 the imaginary part of dielectric function for SiGe alloys with different compositions calculated within DFT-LDA-RPA scheme. In 


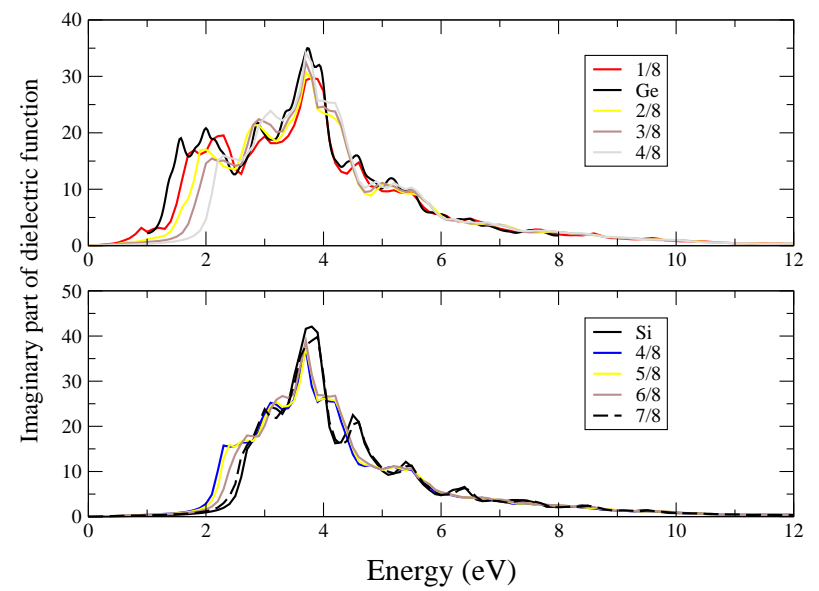

Figure 1 Imaginary part of dielectric function for alloys with different Si compositions calculated within DFT-LDA-RPA scheme. In the upper panel in vicinity of the pure Ge case, in the lower on in vicinity of the pure Si case.

the upper panel in vicinity to the pure Ge case, in the lower in vicinity to the pure Si case. It is clear from this figure that aside from different behaviours at the gap region, induced by the presence of the atoms of the other species in the cell, the spectra both in the Si rich or Ge rich case show rather smooth changes upon varying the concentration. Once the macroscopic dielectric function has been calculated is possible to obtain all the optical functions, for example the real and imaginary part of the refractive index [11]. If one needs to treat properly the one- and two particle-effects in the optical absorption spectra one could use respectively a DFT-GW scheme for self-energy corrected electron-excitation energies and the BSE approach to take correctly into account the electron-hole interaction. The BSE macroscopic dielectric function reads [10]

$$
\varepsilon_{\mathrm{M}}(\omega)=1+2 \lim _{\mathbf{q} \rightarrow 0} v(\mathbf{q}) \sum_{\lambda} \frac{\left|\sum_{v c \mathbf{k}}\left\langle\phi_{v \mathbf{k}^{\prime}}\left|e^{-i \mathbf{q r}}\right| \phi_{c \mathbf{k}}\right\rangle A_{\lambda}^{(v c \mathbf{k})}\right|^{2}}{E_{\lambda}-\omega-i \eta},
$$

where $v(\mathbf{q})$ is the coulombian potential; $\phi_{n \mathbf{k}}$ are the Kohn-Sham wavefunctions; $E_{\lambda}$ and $A_{\lambda}^{(v c \mathbf{k})}$ are the eigenvalues and eigenvectors of the excitonic hamiltonian $\bar{H}^{\text {exc }}$.

In Fig.?? the imaginary part of dielectric function calculated within state of the art techniques which include one-(GW) and two-particle (BSE) effects. Upper panel shows the comparison between theory and experiments after Ref.[16] for pure Si. Lower panel Ge case with experiments after Ref.[17]. In the central panel the theoretical data within DFT-LDA-RPA and GW-BSE relative to the $\mathrm{Si}(50 \%) \mathrm{Ge}(50 \%)$ alloy. It is interesting to note that considering both the one-particle (GW)and the two-particle (BSE) )effects in the 


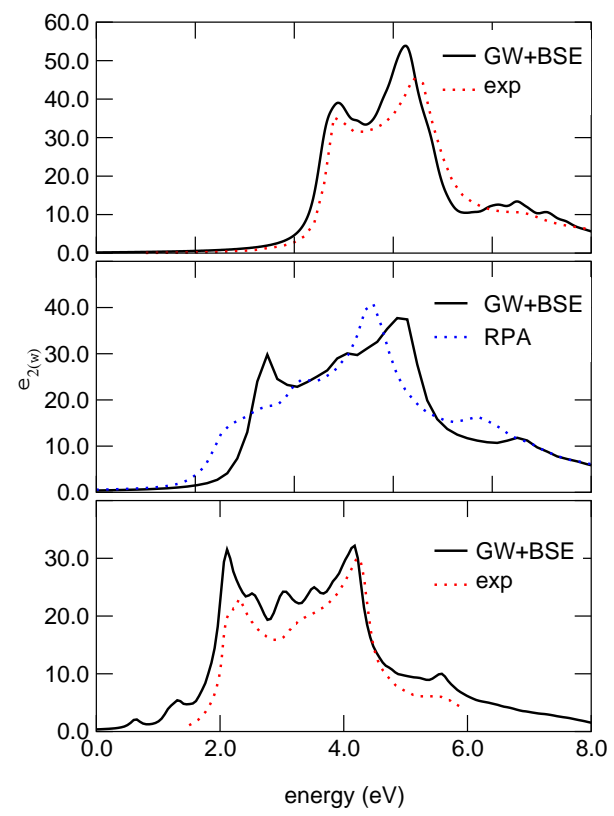

Figure 2 Imaginary part of dielectric function calculated within state of the art techniques which include one-(GW) and two-particle (BSE) effects. Upper panel shows the comparison between theory and experiments for pure Si. In the lower panel Ge case with experiments. In the central panel the theoretical data relative to $\mathrm{Si}(50 \%) \mathrm{Ge}(50 \%)$ alloy.

spectra for pure Si and Ge very good agreement is found with experiments. Infact both the above computational schemes represents state of the art techniques for electronic excitations and optical properties of semiconductors[10]. However the applications of these approaches is limited until now to small cells systems (e.g. in this work two-atoms cells). The results reported in the central panel of Fig. 2 for $\mathrm{Si}(50 \%) \mathrm{Ge}(50 \%)$ show a relatively good comparison between DFT-LDA-RPA and GW-BSE data, aside from the first peak region below $3 \mathrm{eV}$. In comparison with experiments [18], at the optical onset the GW-BSE curve shows good agreement with data obtained at $x=0.4,0.6$ concentrations, while the DFT-LDA-RPA curve suffers of evident mismatch [1]. This point suggests the need of correct inclusion of high-order effects in the Si-Ge alloys optical functions to correctly reproduce their behaviors in the whole energy range.

\section{CONCLUSIONS}

We presented first-principles calculated electronic and optical properties of some SiGe ordered alloys. The ground-state, electronic excitations and optical properties have been calculated within state of the art techniques up to include one- and two-particle effects for the noticeable $\mathrm{Si}(50 \%) \mathrm{Ge}(50 \%)$ case. Comparison with previous experimental and theoretical results is given as possible extensions of optical calculations to more complicated SiGe alloys are discussed. 


\section{ACKNOWLEDGEMENTS}

This work was partially supported by the ENEA Computing environment at Centro Ricerche ENEA Casaccia (Rome). Support from INFM through the PRA project 1MESS is also aknowledged.

\section{References}

[1] R. Ahuja et al., J. Appl. Phys. 93, 3832 (2003)

[2] C. Abstreiter et al., Phys. Rev. Lett.54, 2441 (1985)

[3] T.P. Pearsall et al., Phys. Rev. Lett.58, 729 (1987)

[4] H. Presting, in Challenges for Si-Ge Heteroepitaxy, edited by E. Fitzgerald, J. Hoyt, K.Y. Cheng, and J. Bean, MRS Symposia Proceedings No. 379 (Materials Research Society, Pittsburgh, 1995 pp. 417-432)

[5] B.S. Meyerson, Sci. Am.270, 42 (1994)

[6] B.Y. Tsaur, C.K. Chen, and S.A. Marino, Opt. Eng. 33, 72(1994)

[7] J. Engvall et al., Appl. Phys. Lett.63, 491 (1993)

[8] Douglas Paul, Physics World 13(2), 27-32 (2000)

[9] R. Braunstein, A. R. Moore, F. Herman, Phys. Rev. 109, 695 (1958)

[10] G. Onida, L. Reining, A. Rubio, Rev. Mod. Phys.74, 601 (2002)

[11] G. Cappellini, G. Satta, M. Palummo, G. Onida, Phys. Rev. B 64, 035104 (2001)

[12] U. Schmid, N.E. Christensen, M. Cardona, Phys. Rev. 41, 5919 (1990)

[13] G. Theodorou, P.C. Kelires, C. Tserbak, Phys. Rev. 50, 18355 (1994)

[14] I.N. Remediakis, E. Kaxiras, Phys. Rev. 59, 5536 (1999)

[15] M.S. Hybertsen and S.G. Louie, Phys. Rev. B 34, 5390 (1986).

[16] P.M. Lautenschlager et al., Phys.Rev. B 36,4821 (1987)

[17] D.E. Aspnes and A.A. Studna, Phys. Rev. B 27, 985 (1983)

[18] J.H. Bahng et al. , J.Phys.: Condens. Matter 13, 777-786 (2001) 